Horizons philosophiques

\title{
Hégémonie, fragmentation et mondialisation de la culture
}

\section{Guy Rocher}

Volume 11, numéro 1, automne 2000

L’amodernité de la photographie?

URI : https://id.erudit.org/iderudit/802954ar

DOI : https://doi.org/10.7202/802954ar

Aller au sommaire du numéro

Éditeur(s)

Collège Édouard-Montpetit

ISSN

1181-9227 (imprimé)

1920-2954 (numérique)

Découvrir la revue

Citer cet article

Rocher, G. (2000). Hégémonie, fragmentation et mondialisation de la culture. Horizons philosophiques, 11(1), 125-134. https://doi.org/10.7202/802954ar d'utilisation que vous pouvez consulter en ligne.

https://apropos.erudit.org/fr/usagers/politique-dutilisation/ 


\section{HÉGÉMONIE, FRAGMENTATION ET MONDIALISATION DE LA CULTURE*}

Hégémonie et fragmentation de la culture, un double phénomène, un double mouvement, déjà engagé depuis longtemps et qui ira très probablement en s'accentuant dans ce XX|e siècle qui débute. $\mathrm{Ce}$ n'est évidemment pas en philosophe que je vais en aborder l'analyse, c'est en sociologue. J'adopte donc au départ la définition de la culture que nous en donne l'anthropologie : l'ensemble des représentations, idées, images et comportements qui sont partagés au sein d'une collectivité, qui appartiennent à un temps donné, qui sont également variables d'une société à l'autre, d'une collectivité à l'autre, et qui constituent le miroir, le discours à travers lequel chaque collectivité se perçoit, parle d'elle-même et veut que l'on parle d'elle. Ce qui n'empêche pas que, entendue dans ce sens, la notion de culture rejoigne aussi l'idée de la culture humaniste, celle qui caractérise des personnes dites cultivées, des sociétés cultivées, voire une ère cultivée, c'est-à-dire selon le sens donné à ce terme dans le sillage de la tradition gréco-latine dont nous avons hérité.

\section{Hégémonie et fragmentation : un mouvement oscillatoire}

On peut observer à travers l'histoire une tension constante entre des mouvements d'hégémonie de cette culture et des mouvements de fragmentation, entre certaines formes de domination et même d'impérialisme culturel d'un cóté et, de l'autre, diverses voies de fragmentation, de division, de différenciation. L'histoire de la culture se présente comme une constante oscillation entre des tentatives de domination culturelle et des propensions à la particularisation de la culture. Les exemples historiques sont nombreux. Un des plus intéressants et des plus riches, c'est celui de l'empire romain, des premiers siècles de l'ère chrétienne. Au moment où il recouvrait tout le bassin de la Méditerranée, l'empire romain était dominateur, impérialiste, hégémonique. Du point de vue culturel, l'empire romain avait élaboré des modes de vie, des modes de pensée, des notions

\footnotetext{
* Version abrégée et adaptée d'une conférence prononcée au Collège ÉdouardMontpetit, le 7 avril 2000
} 
d'autorité et de liberté qu'il imposait à tous les peuples qu'il avait conquis. Mais en même temps, l'empire romain connaissait et reconnaissait en son sein des états ou des situations de fragmentation. Les Romains, très habilement, respectaient, par exemple, les droits locaux compatibles avec le leur, les modes de gouvernements locaux, les religions locales. Cet empire, très puissant à l'époque, était en même temps un empire fait de particularités. C'est un des beaux cas que l'on peut relever dans l'histoire de l'hégémonie d'un vaste empire, d'un très puissant empire, et de sa fragmentation. Ce qui apparemment n'était pas le cas d'empires contemporains de cette époque, comme l'empire perse, beaucoup plus unitaire que l'empire romain.

Cette dialectique de l'hégémonie et de la fragmentation n'est pas la même d'un siècle à l'autre, elle peut être plus intense à un moment qu'à l'autre. Nous vivons aujourd'hui une période où ce double mouvement d'hégémonie et de fragmentation est particulièrement intense et rapide. La cause en est le nouveau contexte de la mondialisation dans lequel ce mouvement se produit et qui lui donne son rythme.

\section{Les mondialisations}

Ce qu'on appelle aujourd'hui mondialisation est en réalité fait de trois grandes mondialisations : les mondialisations économique, politique, culturelle. La mondialisation économique est celle dont on parle le plus, celle qui vient d'abord à l'esprit. Mais elle appartient de fait à un vaste système de mondialisation. Elle se présente sous un double aspect. C'est d'abord la mondialisation des lieux de travail : de grandes entreprises ont ouvert des manufactures, des usines à travers le monde, en général dans les pays moins développés. Beaucoup d'entreprises sont maintenant transnationales, elles ont créé un nouveau monde au-dessus des États, au-dessus des nations, un monde industriel qui se déplace. II en a résulté que la division du travail s'est modifiée au cours des dernières années. Une nouvelle division du travail s'est établie entre les pays industriellement développés et les pays moins développés. La main-d'œuvre des pays développés en souffre : elle perd des emplois, cause du chômage. Et les pays moins développés voient leur structure économique et sociale bouleversée et souvent plus affaiblie encore qu'auparavant. L'autre mondialisation économique, c'est la mondialisation du capital, du monde des affaires, l'argent qui circule par milliards : l'argent des banques, des trusts, des caisses de retraite, qui circule sur une plaque mondiale. C'est aussi l'énorme masse d'argent issue du commerce international de la drogue, de la prostitution, de la 
contrebande, du vaste commerce des armes. Ainsi, s'est mis en place un vaste système financier, qui n'est plus transnational, il est ultranational, il est mondial. Et c'est ce système monétaire mondial qui déséquilibre le pouvoir des États nationaux.

Parallèlement à la mondialisation économique, on a assisté depuis le début du $X X X^{e}$ siècle à une mondialisation politique. Se sont créés des rapports politiques qui ne sont plus seulement des rapports internationaux mais qui deviennent des rapports mondiaux. Depuis la Prèmière guerre dite mondiale et surtout depuis la Deuxième, de grands organismes internationaux ont été mis en place, la Société de nations d'abord, puis l'ONU et les multiples autres instances internationales exerçant une autorité sur les États nationaux. Tous ces organismes internationaux développent des normativités, des régulations plus ou moins contraignantes pour les États membres. Un système politique mondial s'est ainsi installé. Aucun État moderne ne peut exercer son autorité sans tenir compte de ce niveau politique, des décisions qui y sont prises, des délibérations qui s'y déroulent, des procès qu'on y intente.

La troisième mondialisation, la mondialisation culturelle, attire de plus en plus l'attention, elle apparaît d'une importance croissante. Elle est en particulier l'objet d'un certain nombre d'études qui sont en cours dans la sociologie américaine autour d'un thème qui s'appelle le "World Polity" en anglais, que je traduis en français par "le diffusionnisme culturel mondial». Les chercheurs rattachés à cette école de pensée ont élaboré la thèse selon laquelle la mondialisation ne se produit pas d'une manière anarchique mais d'une manière de plus en plus organisée, parce qu'au cours des trois derniers siècles, l'Occident, l'Europe d'abord et par la suite l'Amérique, a progressivement répandu et imposé dans le monde entier les institutions et modèles culturels qu'il a longuement produits au cours des siècles précédents. C'est ainsi qu'à travers le monde entier, les systèmes d'enseignement se ressemblent maintenant, parce qu'ils se sont modelés sur le système d'éducation occidental. Même chose pour le droit. Le Common Law des Anglo-saxons et le droit civil des Français, des Allemands et des Espagnols sont devenus les deux formes de droit qui servent de référence obligée dans les différents pays du monde. Et le droit international s'inspire du Common Law et de ce droit civil. De même, les institutions de l'État occidental, sa bureaucratie, son mode d'exercice de l'autorité de l'État, pensés en Occident, se sont répandus dans le monde, principalement sous la 
forme de l'État démocratique, de l'État de droit, dans ses rapports entre l'État et les citoyens. Bref, un ensemble de modèles culturels et d'institutions qui ont eu leur origine en Occident se sont de plus en plus répandus dans le monde entier. Le monde se ressemble de plus en plus. Et cela parce que la majorité des pays de la planète vivent sous l'hégémonie culturelle de l'Occident.

Quels sont les facteurs qui ont joué pour qu'on en vienne à cette domination culturelle? Bien sûr, la colonisation a été un facteur majeur depuis trois siècles. La colonisation par l'Espagne, le Portugal, l'Angleterre, la France, les États-Unis, a été le principal véhicule de diffusion des modèles culturels. Ce fut l'œuvre des militaires, des administrateurs, accompagnés des missionnaires. Surtout depuis la Deuxième guerre mondiale, ce mouvement de diffusion s'est accéléré, avec le changement à la fois et dans les modes de transport et dans les techniques de communication ; il s'est accéléré aussi à la faveur de l'immense nervure que forment les réseaux scientifiques, les réseaux universitaires autour du monde.

La diffusion du modèle occidental est due aussi en grande partie au développement de ce qu'on appelle les organismes non gouvernementaux, les ONG et l'on parle de plus en plus des ONGI, des organismes non gouvernementaux internationaux qui se sont multipliés. De 200 qu'ils étaient en 1900, ils sont plus de 4000 aujourd'hui. On a assisté à une prolifération, une inflation de ces mouvements et organismes non gouvernementaux. Et ceux-ci ont servi et servent de courroie de transmission d'une grande partie de la culture occidentale. Tout en servant à l'occasion des intérêts particuliers, les ONGI contribuent à l'hégémonie culturelle occidentale.

Mais ce qui fait l'originalité de la thèse de cette école de pensée, c'est l'idée que la mondialisation culturelle entendue en ce sens a pavé la voie aux deux autres mondialisations, l'économique et la politique. C'est parce que l'Occident a diffusé et imposé ses institutions et ses modèles dans le monde entier, notamment ceux de l'État et du monde du travail, que l'unité politique peut se réaliser sur le plan international et qu'un vaste marché industriel et financier a pu se mettre en place. Les mondialisations économique et politique ont donc pris appui sur les institutions et modèles culturels diffusés à partir de l'Occident. Sans l'hégémonie culturelle occidentale sur le monde, celui-ci serait encore divisé en civilisations ou en blocs étanches et irréductibles les uns aux autres. Les chercheurs qui se rattachent à cette école s'emploient à faire la preuve de cette 
hypothèse à travers une série d'études quantitatives publiées récemment.

On ne peut que trouver cette thèse - ou cette hypothèse fascinante. Elle apporte une explication originale au procès de mondialisation en cours. Une explication qui vient relativiser l'explication exclusivement économique que l'on donne couramment de la mondialisation. Si le marché international est en voie d'installer une hégémonie économique, c'est qu'il a été précédé d'une hégémonisation culturelle en marche depuis trois siècles.

Cette thèse mérite qu'on continue de l'explorer : elle me paraît fertile. À la condition cependant de la compléter par un certain nombre de considérants que les promoteurs de la thèse n'explicitent pas toujours suffisamment. II faut d'abord clarifier le rôle des récepteurs locaux dans ce mouvement de diffusion, le rôle des acteurs, institutions, groupes et mouvements sociaux locaux qui ont reçu les innovations culturelles, les ont accueillies, les ont intégrées dans les structures et la vie de leur collectivité. Si l'on néglige cette dimension, la thèse de la diffusion prend un caractère mécaniste, laissant croire que la réception des institutions importées allait de soi, se réalisait d'une manière automatique. Au surplus, il faut tenir compte des adaptations que ces acteurs locaux ont faites des modèles culturels occidentaux. Ceux-ci, il est vrai, se sont répandus dans le monde, mais avec des adaptations en Afrique, en Asie, en Polynésie. Non seulement des acteurs les reçoivent-ils, mais ils les adaptent et les retravaillent. La thèse américaine ne me paraît pas très claire là-dessus. J'ajoute que l'hypothèse de la mondialisation culturelle oublie un peu trop les nombreuses résistances que cette mondialisation culturelle a rencontrées : résistances régionales, nationalistes, tribales, économiques, politiques et culturelles. En conséquence de ces résistances, on a pu observer très souvent que les modèles culturels occidentaux ont été plaqués artificiellement et superficiellement sur la réalité de bien des pays. Les systèmes d'éducation et les systèmes juridiques importés par les pays colonisateurs ont dû et doivent encore - vivre avec des mœurs, des institutions, des droits locaux et chercher des formes de pluralisme d'accueil pour éviter de n'être que superficiellement installés en pays étranger.

Ces considérants sont importants pour éviter que cette thèse ou cette hypothèse - ne soit qu'une vision américaine, une vision yankee, de ceux qui sont situés au cœur, dans le noyau dur de l'hégémonie culturelle et qui ont de la difficulté à percevoir les conditions de 
réception des apports culturels et les résistances que des cultures et institutions locales leur opposent.

\section{La culture de l'entertainment}

Un autre volet de l'hégémonie culturelle - le plus visible peut-être - se cristallise sous la forme de la diffusion de la culture de l'entertainment. La culture de l'entertainment, c'est la culture que l'on peut appeler populaire, celle qui est portée par tous les médias modernes, le cinéma, la radio, le disque, les revues, les magazines et par le monde matériel qui entoure la diffusion culturelle : l'ensemble des biens de consommation. Cette diffusion se fait par la voie d'un grand canal, partout le même à travers le monde, la langue anglaise, aujourd'hui identifiée à cette culture de l'entertainment. La culture de l'entertainment est différente des grands modèles culturels, en ce sens qu'elle est récente et surtout qu'elle est le fait de l'hégémonie d'un pays, les États-Unis. À travers la culture d'entertainment, c'est la culture yankee, produit USA, qui se répand et se diffuse dans le monde. Un historien et sociologue américain, George Ritzer, a fait l'analyse de cette culture de l'entertainment sous le vocable de "la macdonalisation de la société", dans son livre The Macdonalization of Society. La restauration MacDonald est en quelque sorte l'archétype d'une culture fast food, l'archétype d'une culture qui insiste sur la rationalisation technique de tout ce qui entoure notre vie, pour faire tout vite et facilement, du tout-prêt à consommer. C'est la rationalisation de la nourriture fast food, du vêtement, de l'habitation, du transport et finalement l'homogénéisation de tout.

\section{L'hégémonie de la culture juridique}

Je voudrais analyser un autre aspect de l'hégémonie culturelle, mais sur un autre plan que celui de la mondialisation, c'est celui de l'hégémonie de la culture juridique plus particulièrement caractéristique de la civilisation occidentale contemporaine. Nous vivons, surtout depuis la Deuxième guerre mondiale, une époque où le consensus social est de plus en plus fragile, qui résulte de la relativisation de la morale de l'éthos collectif, des valeurs. En conséquence, il se trouve que le droit est le seul lieu où survit un certain consensus social. Les grands principes que le droit est allé emprunter à la philosophie morale, à l'éthique, à la philosophie politique, c'est dans les Chartes des droits et libertés de la personne qu'on les retrouve. Celles-ci sont devenues notre nouvelle Bible. Ces chartes 
représentent ce que j'appelle la culture juridique qui remplace la culture morale. Aujourd'hui, les normes de conduite et même les normes morales se pensent dans la logique du droit, dans la rationalité du droit. II faut faire un grand effort pour penser moralement en dehors du droit. C'est ainsi que les guides de déontologie, les guides d'éthique récemment développés, on les appelle instinctivement des "codes" d'éthique ou de déontologie. La mentalité juridique, qui s'est instaurée dans notre monde occidental, est maintenant en train de faire partie de la domination culturelle mondiale, elle fait partie du modèle culturel diffusé à travers le monde.

Cette culture juridique est importante : c'est elle qui nous définit comme citoyen, mais c'est même la rationalité juridique qui nous définit maintenant comme être humain, c'est-à-dire comme sujet de droit, tout comme c'est selon elle que se définit la structuration de nos relations sociales. Le droit est envahissant, il exerce une emprise croissante sur nos vies, nos institutions, nos relations humaines, nos sociétés. Le droit s'insère partout; et depuis 50 ans il a proliféré d'une manière extraordinaire. Les lois et les règlements publics et privés se multiplient et, plus encore, la jurisprudence construite sur ces lois croît de manière inflationnaire tandis que ce qui s'appelle "la doctrine" juridique, c'est-à-dire les travaux savants sur le droit, remplit des bibliothèques. L'hégémonie de la culture juridique s'appuie donc sur une production législative et judiciaire inouie.

L'hégémonisation de la culture juridique s'explique en grande partie par suite de la coïncidence aisément observable entre les valeurs qu'elle porte et celles de la classe moyenne. Au cours du XXe siècle, la classe moyenne est devenue la classe dominante, presque la seule classe sociale existante. La classe agricole et l'aristocratie ont fondu, la classe ouvrière se rétrécit comme peau de chagrin, nous accédons tous à la classe moyenne. Or c'est cette vaste classe moyenne qui est la porteuse de la culture juridique parce qu'il y a une coïncidence, presque une complicité, entre la mentalité de la classe moyenne et la culture juridique. La culture juridique est individualiste, comme l'esprit de la classe moyenne. Elle privilégie avant tout la personne, ses droits, ses responsabilités; c'est le monde de la classe moyenne. La culture juridique met l'accent sur la propriété, les biens, comme la culture de la classe moyenne. Elle met l'accent sur la liberté de la personne, elle favorise l'initiative de l'entrepreneur. Toute la culture juridique s'est ainsi insérée dans la culture de classe moyenne, elle est l'expression de la classe moyenne, et c'est cette dernière qui assure l'hégémonie de la culture juridique. 


\section{Les fragmentations}

J'ai accordé une grande importance à différentes formes d'hégémonie culturelle, parce que je crois qu'elles ne sont pas toujours apparentes ou encore qu'elles ne sont pas toujours analysées dans le détail. L'hégémonisation ne doit cependant pas occulter les mouvements de fragmentation qui l'accompagnent, lui sont parallèles ou viennent la contrer.

L'empire de la classe moyenne que je viens d'évoquer engendre ses propres fragmentations culturelles et institutionnelles. Je voudrais évoquer celles qui me paraissent particulièrement significatives. Un des traits dominants de la société de la classe moyenne, c'est la professionnalisation du monde de l'emploi et par conséquent la fragmentation des cultures professionnelles. La société contemporaine n'est plus faite principalement de classes sociales; elles se sont en quelque sorte éliminées d'elles-mêmes. La société moderne est faite de professions de plus en plus nombreuses. Le modèle des deux grandes professions dites libérales d'il y a quelques années, la médecine et le droit, ce modèle a été assimilé et étendu à tout un ensemble de métiers et de lieux de travail. Les professions se sont multipliées et continuent à le faire : presque tous les emplois aspirent à se dire professionnels. Or, chaque profession a développé sa culture. Chaque profession a son mode de pensée, son idéologie, sa représentation, ses rituels. Chacune de ces cultures professionnelles tire sa source d'une formation commune au collège, à l'université, devenus les creusets de nouvelles cultures professionnelles. La société professionnelle est ainsi de plus en plus diversifiée. Cette fragmentation des cultures professionnelles, une fragmentation profondément sociale, est devenue un des phénomènes dominants de la société contemporaine.

La multiplication des cultures professionnelles prend également sa source dans une autre forme de fragmentation qui lui est complémentaire et qui la renforce, la fragmentation du savoir. Depuis le début du XXe siècle, l'inflation et la fragmentation du monde du savoir est un des phénomènes les plus marquants de la culture mondiale contemporaine. Au début du siècle, on parlait des sciences sociales et même au singulier de la science sociale. Après quelques années, on a commencé à diversifier la science sociale, et à distinguer l'économique, la politique, la sociologie. Maintenant, les sciences sociales sont infiniment diversifiées et chacune d'elles compte des sur-spécialités. On ne fait plus de la sociologie, on fait de 
la sociologie politique, de la sociologie économique, de la sociologie de la famille ou des religions, etc. II n'y a plus une sociologie, mais des sociologies. De même pour chacune des autres disciplines des sciences sociales et humaines, cet éclatement du savoir accompagne précisément la prolifération des professions. Chacune des professions s'insère dans un savoir de plus en plus spécialisé.

Fragmentation professionnelle et fragmentation du savoir : deux phénomènes qui ouvrent la voie à ce que, dans le langage étatsunien plutôt que canadien, on appelle le multiculturalisme. L'analyse postmodernisme a voulu mettre en lumière le fait que la société a maintenant perdu l'unité fonctionnelle et organique qu'elle a pu avoir : elle est maintenant composée de la juxtaposition de communautés et de groupes culturels ayant chacun leur spécificité et leur irréductibilité. Or, ce multiculturalisme à l'américaine se matérialise sur le plan politique par la montée en nombre et en force des groupes d'intérêt et des groupes de pression. Chacun de ces groupes s'emploie à promouvoir les intérêts particuliers d'un segment de la société. Des leaders émergent qui en deviennent les porte-parole. Certains de ces groupes sont assez nombreux, puissants et organisés pour avoir leur propre bureaucratie de "permanents". À la démocratie de la majorité se substitue progressivement une démocratie des minorités, très certainement une démocratie des groupes d'intérêt et des groupes de pression. On observe ici la fragmentation la plus fondamentale de la société contemporaine.

J'ajoute un dernier facteur de fragmentation, le mouvement de sécularisation propre à l'Occident. On associe rarement sécularisation et fragmentation sociale. Dans l'histoire de l'humanité, et peut-être surtout du monde occidental, les religions ont joué un double rôle, de rassembleur et de diviseur. Les religions ont créé des Nous communautaires très forts en même temps que des clivages profonds entre le Nous et tous les Autres qui ne sont pas élus. II s'agissait moins de fragmentation que de grandes divisions sociales. Cette division demeure aujourd'hui, si l'on fait référence au fossé entre l'Islam et le monde judéo-chrétien. Mais parallèlement à cette rupture, la sécularisation apporte une nouvelle fragmentation.

Dans son sens le plus étendu, la sécularisation fait référence tout à la fois à la séparation de l'Église et de l'État, à la marginalisation et mise à l'écart du sacré, à l'éclipse des pouvoirs ecclésiastiques sur l'État et dans la société. À travers ces diverses manifestations, la 
sécularisation ne signifie pas la prédominance de l'athéisme, elle désigne plutôt l'éclatement des croyances religieuses, l'acceptation du pluralisme religieux et, par conséquent, le retrait de la religion de la sphère publique. L'ancien consensus religieux se dissipe pour laisser place à la diversité des croyances et pratiques religieuses et à l'individualisation de la religion. Vue sous cet angle, la sécularisation du monde moderne en devient un puissant facteur de fragmentation.

\title{
Conclusion
}

L'historien américain Eugen Weber, spécialiste de l'histoire des campagnes françaises, a intitulé un livre-choc La fin des terroirs. La question se pose aujourd'hui d'une manière qui peut être dramatisée : l'hégémonisation culturelle du monde entraîne-t-elle en effet la fin des terroirs? On sait déjà que nous assistons à la disparition d'un grand nombre de langues, de cultures locales et régionales, de droits coutumiers, de traditions, de rites et de coutumes. Mais en même temps, nous assistons à une renaissance et à une revalorisation de divers éléments des patrimoines régionaux et nationaux. La dialectique se poursuit entre une culture mondiale qui semble s'imposer d'elle-même et les particularismes historiques qui ont fait la riche diversité des cultures multiples. La fragmentation, d'une certaine manière, favorise la vie et la survie de ces particularismes. Elle vient de la sorte contrer, ralentir et peut-être neutraliser le vaste procès d'homogénéisation culturelle que le monde connaît aujourd'hui et que la technologie moderne favorise plus que jamais. On peut prévoir, pour le XXle siècle, une dialectique entre l'hégémonisation et la fragmentation encore plus vive, plus animée, plus intense que celle dont le $X X$ siècle fut témoin.

\author{
Guy Rocher \\ Université de Montréal
}

